\title{
Experimental Investigation on the Deformation Response of Hybrid 3D Woven Composites
}

\author{
Mark Pankow* and Chian-Fong Yen* \\ ARMY Research Laboratory, Aberdeen Proving Ground, MD, 21005, USA \\ Miranda Rudolph, Brian Justusson, Dianyun Zhang and Anthony M. Waas ${ }^{\dagger}$ \\ Aerospace Engineering, University of Michigan, Ann Arbor, MI, 48109
}

\begin{abstract}
This paper presents results of an experimental investigation on the tensile response of "hybrid" 3D woven composites (H3DWC), where "hybrid" refers to different constituent fibers, including carbon, glass, and kevlar that are integrally woven into a single preform. The H3DWCs are made through a $3 \mathrm{D}$ textile weaving process. Five different versions of hybridized architectures were examined to determine the progression of failure under tensile loading. The different types of H3DWC were compared against one another to understand the benefits of hybridization and the resulting performance enhancements.
\end{abstract}

\section{Nomenclature}

\author{
VARTM Vacuum Assist Resin Transfer Mold \\ ENF End Notch Flexure \\ $3 D W C \quad$ Three Dimensionally Woven Composites \\ DIC Digital Image Correlation
}

\section{Introduction}

With the widespread use of composite materials in both aerospace and land vehicle structures, and other industrial sectors (such as in wind energy systems), a detailed understanding of deformation response and failure is needed for proper mass implementation. Typical tape based laminated composites offer high performance, particularly in stiffness properties, but the delamination mode of failure can greatly reduce structural integrity. This mode of failure is critical to mass implementation of composite materials and it is beneficial to limit and/or control the delamination mode of failure. Various techniques have been developed, such as stitching and z-pinning to provide through-the-thickness reinforcements. 3D weaving has shown great promise by using mechanical binding through the thickness to prevent delamination from occurring and propagating rapidly through the material. This technology came from the industrial revolution where looms were used to make textiles and use of the looms requires little alterations from their original manufactured state. Integral woven materials, known as preforms, can be woven in one process to include a series of weft fibers with a reinforcing fiber. The preform is subsequently infused with a matrix material using a Vacuum Assist Resin Transfer Molding (VARTM) process to create a solid structural panel, that can be manufactured to net shape. 3D woven composites have been shown in previous studies to have increased mechanical performance ${ }^{1}$ and the ability to prevent the spread of delamination or completely eliminate this mode altogether $^{23}$. This paper will focus on H3DWC, where the hybridization comes from the use of different types of fibers in the same architecture. Hybridization can result in a number of increases in mechanical performance including increased energy absorption ${ }^{45}$ and improved tensile properties characterized by increases in failure strain. ${ }^{6}$ Hufenbach et. al. have shown hybrid 3DWC can be tailored for specific structural performance. ${ }^{7}$

\footnotetext{
*Army Research Laboratory, Aberdeen Proving Ground, MD, 21005

${ }^{\dagger}$ Felix Pawlowski Collegiate Professor, Aerospace Engineering, University of Michigan, Ann Arbor, MI, 48109. Fellow AIAA
} 
Hufenbach was able to localize the delamination mode using different fiber combinations. The authors assertion is that hybridization of the 3DWC structure could be tailored for the specific need of the structure by varying the fiber type and weave architecture. In this investigation carbon, glass, and kevlar fibers were examined in different preforms that have distinct hybrid architectures. The carbon fibers are used because of their strength and stiffness, the glass for its relatively low cost (high strength per unit cost) and kevlar for its high resistance (characterized by its ductility) to failure. The combination of the three types of fibers has been investigated to determine the mechanical response of the combined hybrid 3D woven composite.

\section{Material}

The main difference between laminated composites and 3DWC is the inter-plane reinforcement through fiber weaving. The binding fiber tows bridge the multiple layers and bind them together. The material that will be focused on in this study is the Z-fiber architecture, also referred to as an orthogonal architecture in the $3 \mathrm{D}$ textiles literature.

The Z-fiber architecture has a very rigid series of warp and weft fibers (the term fiber and fiber tow will be used interchangeably throughput this discussion) that are maintained in-plane through out the panel with little to no undulation due to the tension applied to the fibers during weaving. A set of fibers running in the direction of the warp fibers is drawn from the bottom to the the top to bind all the layers together, and is usually inserted in-between the spaces of the warp fibers. Figure 1 shows one of the possible paths that the Z-fiber could follow in the weaving process. This path is shown in yellow and binds all the layers through the thickness together, however there are multiple different paths that can be formed creating different types of architectures such as two binding fibers, or weaving through every other layer depending on the desired volume fraction of the binder yarn.

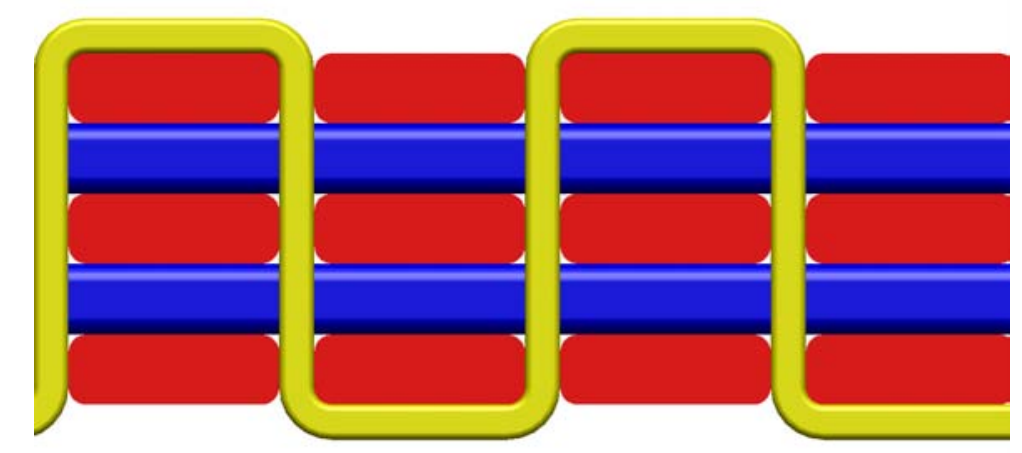

Figure 1. The yellow color path represents the course that the Z-fiber follows during the weaving process.

Five different architectures have been investigated to determine which configurations show the highest degree of improved hybridized mechanical performance characterized by their tensile response. The first architecture, termed the baseline, has the same orthogonal architecture as the hybrid panels, however, has no hybridization, that is, the preform is made entirely of only S-2 glass tows. The next variation is an unsymmetric architecture that consists of two layers of orthogonally oriented in-plane carbon and the remainder glass. The scaling of this architecture is investigated through two panels that have different thicknesses. A symmetric architecture is investigated to understand the hybridization effect on both outer surfaces, much like a "sandwich panel". The final architecture features functionally graded material where the carbon layers are removed through the thickness in a graded manner. The details of the architectures can be found in figure 2, which provides some details of the Z-fiber path. After these preforms were manufactured they were infused with an SC-15 polymer matrix material using a VARTM process. Material Properties for 
each constituent are provided in table 1 as a reference.

Table 1. Material Characteristics for fibers and matrix used.

\begin{tabular}{ccccccc} 
& $\begin{array}{c}E_{1} \\
\text { msi }\end{array}$ & $\begin{array}{c}E_{2} \\
\text { msi }\end{array}$ & $\begin{array}{c}\nu_{12} \\
-\end{array}$ & $\begin{array}{c}\nu_{23} \\
-\end{array}$ & $\begin{array}{c}G_{12} \\
\text { msi }\end{array}$ & $\begin{array}{c}G_{23} \\
\text { msi }\end{array}$ \\
\hline Carbon & 40.03 & 2.50 & 0.2 & 0.25 & 2.70 & 0.70 \\
\hline Glass & 16.56 & 16.56 & 0.22 & 0.22 & 6.74 & 6.74 \\
\hline Kevlar & 16.24 & 16.24 & 0.36 & 0.36 & 8.12 & 8.12 \\
\hline SC-15 & 0.360 & & 0.35 & & & \\
\hline
\end{tabular}

Macroscopic images of the samples along with close-up images after the resin was infused have shown possible differences that exist in the architectures and also imperfections that exist in the matrix material. Figure 3 shows the architectures. A few comments should be made at this point with respect to the unsymmetric architecture shown. The carbon fiber tows are at the top of the specimen, which can be seen as the black tows. These materials were fabricated by a VARTM process. The drawing of the matrix material through the preform with a vacuum can leave trapped air bubbles in the specimens. These air bubbles are termed voids. There seems to be a wide variation between samples.

Table 2. Architecture thicknesses and layer breakdown. The functionally graded sample is based on effective carbon layers

\begin{tabular}{ccccc} 
& Thickness (in) & \# of Layers & \# of Carbon Layers & \% Carbon \\
\hline Baseline & 0.340 & 9 & 0 & 0 \\
\hline Thin Unsymmetric & 0.346 & 9 & 4 & 44.5 \\
\hline Thick Unsymmetric & 0.659 & 17 & 4 & 23.5 \\
\hline Thick Symmetric & 0.626 & 17 & 7 & 47.0 \\
\hline Functionally Graded & 0.649 & 17 & 41.2 \\
\hline
\end{tabular}

The unsymmetric samples, as cured, will experience different thermal strains during the curing process and will cause minor bending in the samples. These initial curvature can be estimated using lamination theory as shown in the Appendix. To characterize the initial curvature, samples were cut into 6in $x$ 6in square panels, and the surface elevation of the sample was measured for its "out of flatness". For the thin panels, the radius of curvature in the two principal directions were approximately in the range of 500-600 inches $(15.24 \mathrm{~m})$. A characteristic surface elevation plot is shown in figure 4 . 


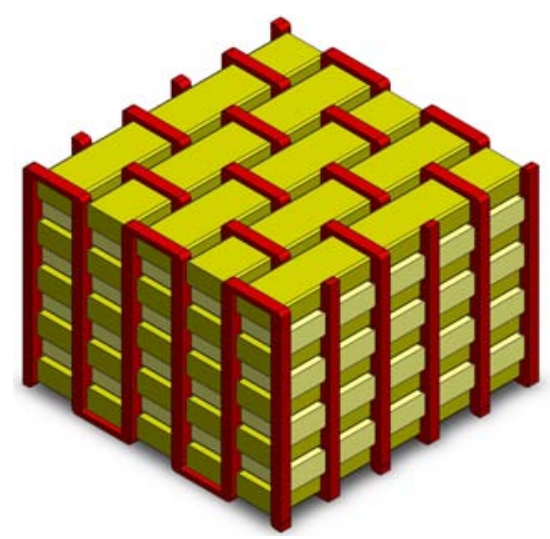

(a) Baseline architecture - The Z-fiber for these samples is glass just shown as kevlar to differentiate it from the rest of the glass fiber tow bundles.

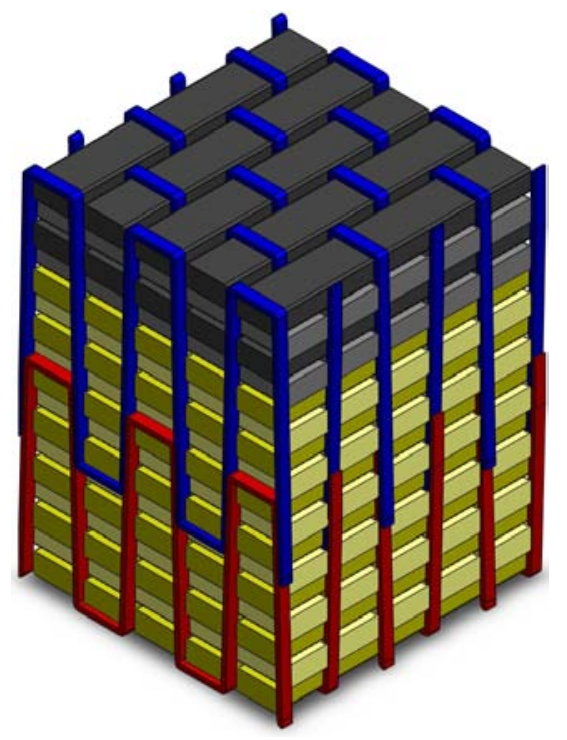

(c) Thick Unsymmetric

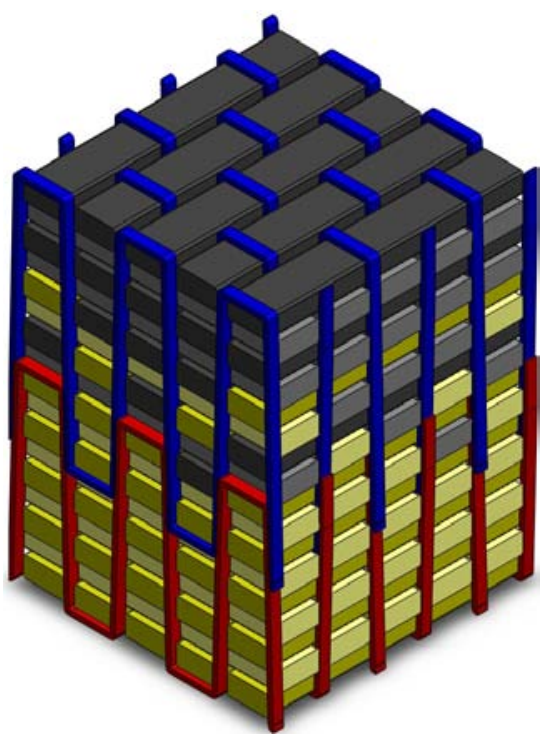

(e) Functionally Graded

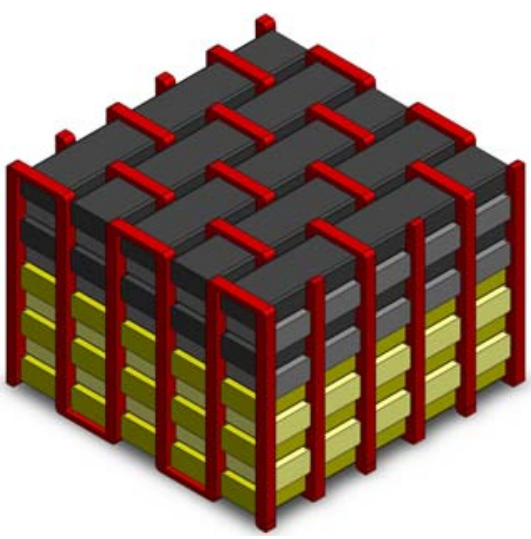

(b) Thin Unsymmetric

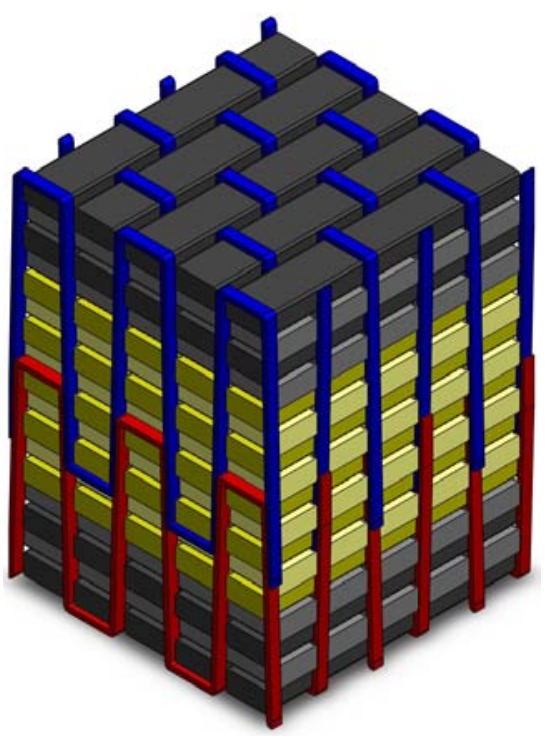

(d) Thick Symmetric

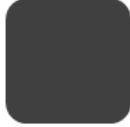

Carbon

Glass

Kevlar

(f)

Figure 2. H3DWC architectures investigated 


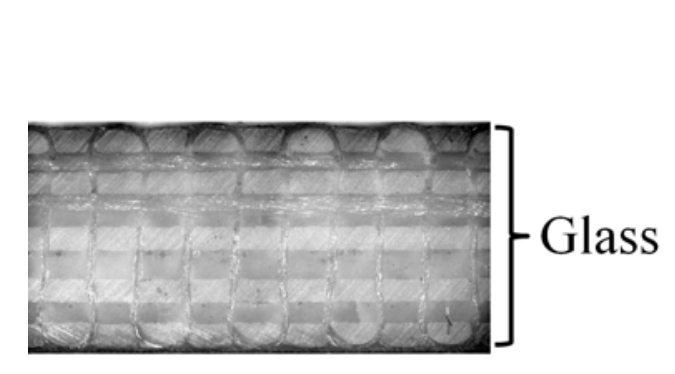

(a) Baseline

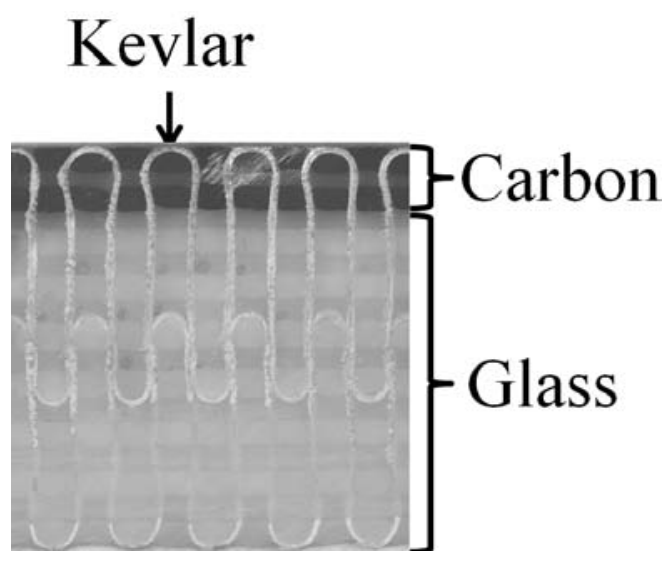

(c) Thick Unsymmetric

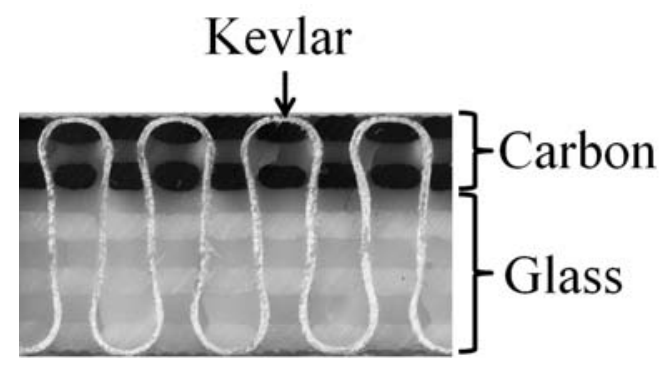

(b) Thin Unsymmetric

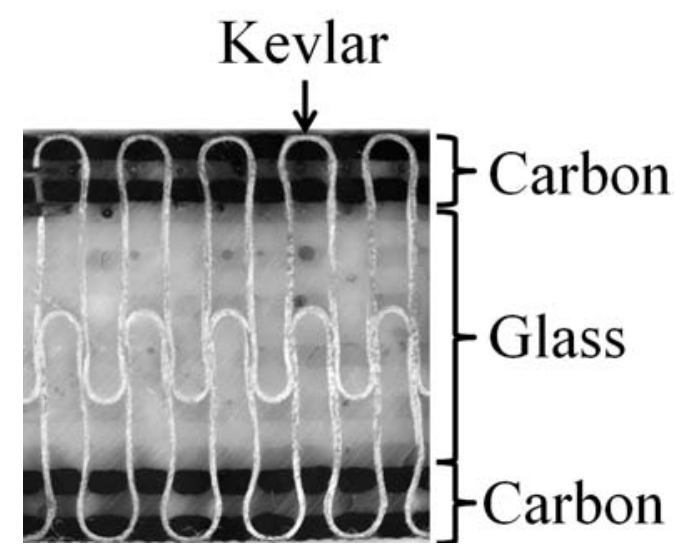

(d) Thick Symmetric

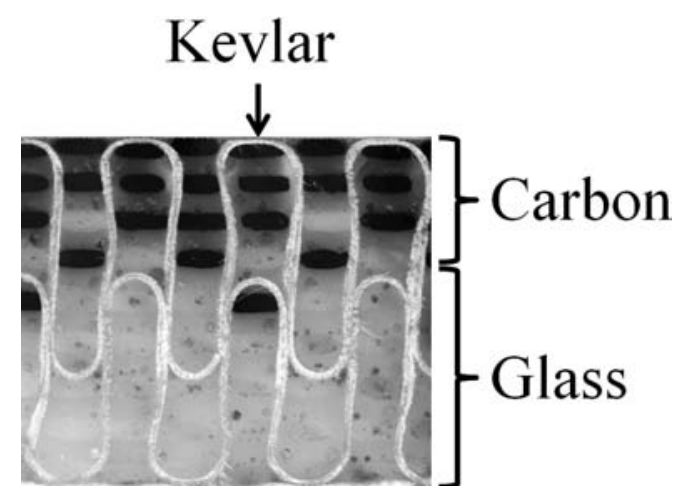

(e) Functionally Graded

Figure 3. Polished surfaces of $3 \mathrm{D}$ woven samples after resin infusion.

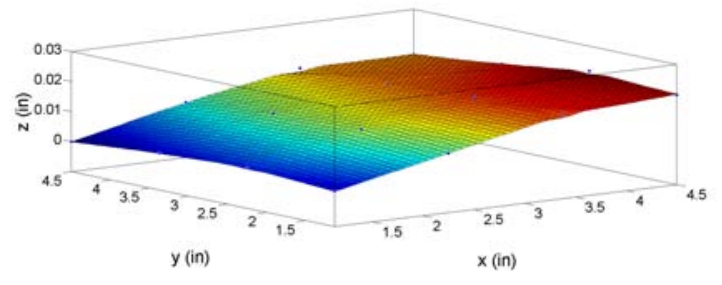

(a)

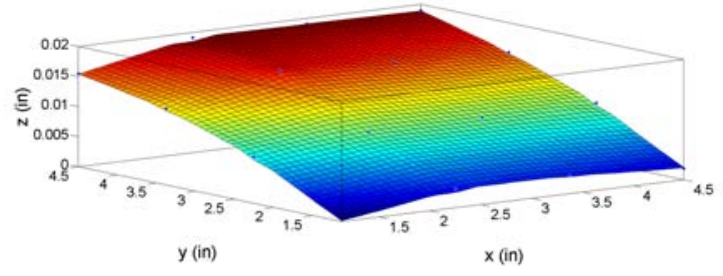

(b)

Figure 4. Curvature plots for unsymmetric samples 


\section{Tension Tests}

Tension tests were performed to determine the elastic response of the panels along with the strength of the 3DWCs. Dogbone tension samples were cut in the two main fiber directions, warp (weaver) and weft (fill) using a water jet cutting machine. These two directions were examined to determine the effective in-plane properties. Samples were tested in a hydraulically activated load frame in a quasi-static (0.0004 in/sec) manner to try to resolve the progression of failure in the samples. Images of speckled surfaces were taken every 5 seconds using two 12 Megapixel cameras to image the front and back surfaces at the same time and an additional third 5 Megapixel camera to image one side face of the sample. These images were analyzed using digital image correlation (DIC) software to determine the strain response on the surface of the material. The strain contours were then used to determine the effective average strain in the sample by taking the average over the surface area that would correspond to a strain gage (local) or the global strain (entire gage section).

Effective stress-strain relationships, based on grip loads and gage cross sectional area, and local (and global) strains were determined from the measurements. From these relationships, the effective moduli, failure strengths and failure strains were determined. The effective moduli for the different architectures can be seen in table 3 , it should be noted here that the moduli for the unsymmetric samples have different surface strains (carbon side vs. glass side) as a result of the hybridization and because of deformation asymmetry resulting in bending. For this reason, the effective modulus is that which corresponds to the stress vs. average strain (average of the carbon side and glass side), and this is used throughout. The Appendix provides a comparison between CLPT based effective moduli and those that were measured.

Table 3. Effective Tensile Modulus

\begin{tabular}{ccc}
\hline \multirow{2}{*}{ Baseline } & \multicolumn{2}{c}{$(\mathrm{Msi})$} \\
\cline { 2 - 3 } & Warp & 2.83 \\
\hline \multirow{2}{*}{ Thin Unsymmetric } & Weft & $3.25 \pm 3.0 \%$ \\
\cline { 2 - 3 } & Warp & $4.61 \pm 11.34 \%$ \\
\hline \multirow{2}{*}{ Thick Unsymmetric } & $5.00 \pm 3.58 \%$ \\
\cline { 2 - 3 } & Warp & $3.7 \pm 13.78 \%$ \\
\hline \multirow{2}{*}{ Thick Symmetric } & Weft & $4.79 \pm 5.47 \%$ \\
\cline { 2 - 3 } & Weft & $6.054 \pm 11.34 \%$ \\
\hline \multirow{2}{*}{ Functionally Graded } & Warp & $4.47 \pm 18.99 \%$ \\
\cline { 2 - 3 } & Weft & $5.33 \pm 0.4 \%$ \\
\hline
\end{tabular}

The hybridization effects can be seen with an increase in effective axial stiffness as an effect of the addition of the carbon in comparison to the baseline orthogonal weave. The increase in stiffness scales with the amount of carbon and this is to be expected because of a simple "rule of mixtures" analysis that is adequate for the axial deformation (see also, Appendix). Additionally, for these materials the weft direction is stiffer than the warp direction due to the fact that there is one additional fiber tow layer in the weft direction. For these architectures, the weft direction tows will be on the surface of each material. The effective stress-strain response can be seen in figure 5, which compares each of the architectures for the two major orientations in each panel.

The stress-strain responses show two phenomena, the first is that the weft direction results show a higher degree of linearity. This is due to the fact that loading occurs directly on the fiber tows, situated on the outer most surface. The second is that the warp direction shows more of a progression in failure of the material and subsequent deviations from linearity relatively early in the loading regime. This occurs because the surface will show matrix micro cracking occurring and creates and effective softening of the material. The architectures show an increasing effective modulus as a function of the amount of carbon, with the thick symmetric panel having the highest percentage of carbon. The effects of hybridization can be seen from these effective responses, and most notably, in the ultimate strength of the material. The strength of the individual 


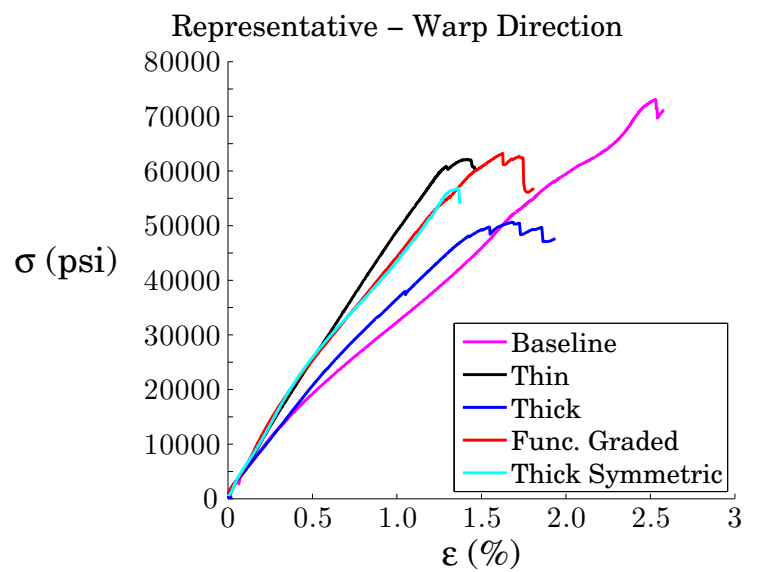

(a) Warp

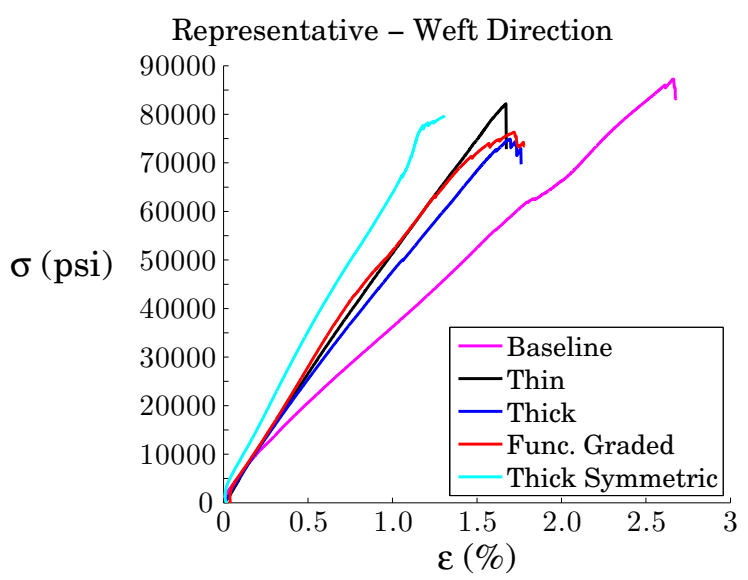

(b) Weft

Figure 5. Comparison of Stress vs. Strain responses for the different H3DWC

fibers are, S2-Glass has a tensile strength of approximately $709 \mathrm{ksi}$ and IM7 has a tensile strength of $747 \mathrm{ksi}$. Since there is very little difference in strength it is expected that there will be minimal increase in strength from the hybridization. However, due to the fact that the different fiber tows will have different stresses at the same externally applied displacement indicates that the carbon may fail earlier than the glass. The ultimate strength of the material has been reported in table 4, which shows that the pure glass architecture out performs the other configurations in terms of maximum load. The carbon layers fail before the glass layers have achieved their maximum load carrying capability. The fact that the hybrid panels have stress gradients through the panel thickness suggests that the response of the hybrid architectures requires further study, particularly their flexural response.

Table 4. Ultimate Tensile Strength

\begin{tabular}{ccc}
\hline \multirow{2}{*}{ Baseline } & \multicolumn{2}{c}{$($ ksi $)$} \\
\hline \multirow{2}{*}{ Thin Unsymmetric } & Warp & $77.23 \pm 5.2 \%$ \\
\cline { 2 - 3 } & Weft & $86.84 \pm 0.91 \%$ \\
\hline \multirow{2}{*}{ Thick Unsymmetric } & Weft & $80.205 \pm 4.91 \%$ \\
\cline { 2 - 3 } & Warp & $60.51 \pm 10.84 \%$ \\
\hline \multirow{2}{*}{ Thick Symmetric } & Weft & $77.01 \pm 5.90 \%$ \\
\cline { 2 - 3 } & Weft & $68.07 \pm 8.70 \%$ \\
\hline \multirow{2}{*}{ Functionally Graded } & Warp & $71.48 \pm 21.38 \%$ \\
\cline { 2 - 3 } & Weft & $72.55 \pm 10.47 \%$ \\
\hline
\end{tabular}

As mentioned previously, the effective moduli were calculated from the DIC measurements taken based on the average strains from both the glass side and the carbon side. As shown in the Appendix, the effective moduli involve not only individual layer stiffnesses but also the various volume fractions. This data will now be looked at in further detail. The strain-time history response for the front, back, and side of the unsymmetric hybrid sample is plotted in figure 6 . This plot clearly shows that the carbon and glass samples experience different axial strains caused due to two reasons. The first is that the samples are initially curved. This initial curvature creates an initial straightening of the sample that can lead to straining asymmetry. This effect is highlighted from some of the samples which showed initial compression upon loading as seen 
in figure 7. During the curing process, because of different coefficients of thermal expansion, the carbon tows have a longer effective length and thus during bending the outer carbon surface undergoes compressive straining. This point is schematically illustrated as in figure 8 .

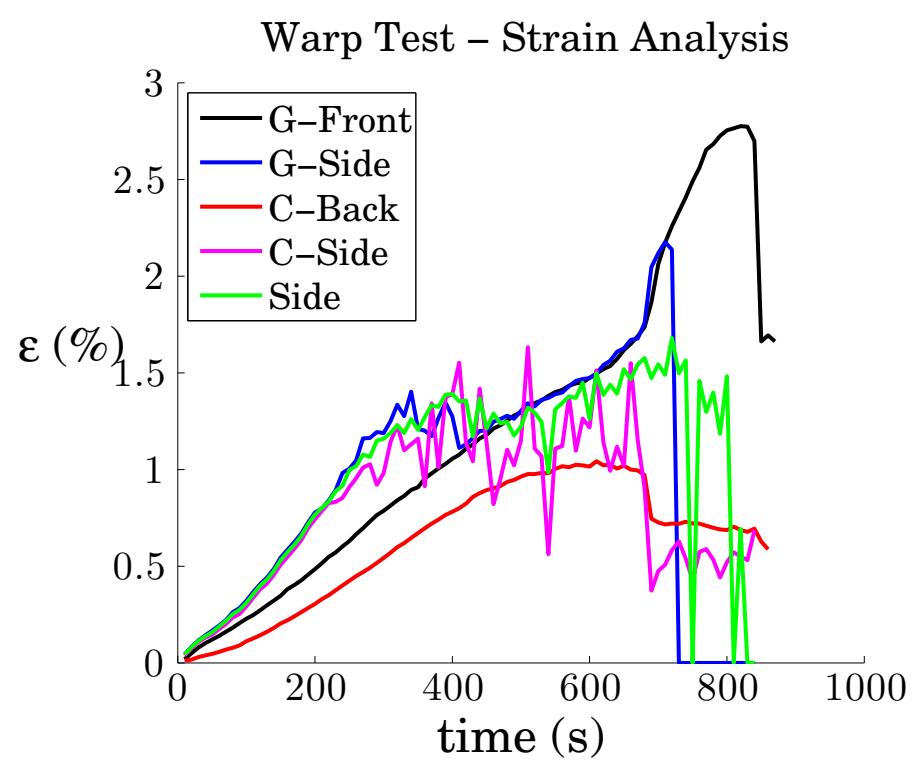

Figure 6. Strain vs. time for composite hybrid samples, comparing the strains that occur on the different surfaces of the material.

The second reason for the difference in surface strains is due to the distribution of load in each layer. After the samples are "straightened", during loading, the stiffer carbon fibers will carry a larger percentage of the load than the glass layers. As carbon tows reach a critical strain near rupture, the glass fiber tows begin to take a proportionally larger amount of strain. Once the carbon tows have failed, the surface strains on the glass side begin to have a similar strain contour behavior corresponding to the baseline architecture, which is a pure glass panel. This response shows how the H3DWC is responding to the sudden stress redistribution conditions at the fiber tow scale, and also shows that carbon fiber tow failure does not result in catastrophic failure of the panel since the load is still carried by the glass fiber tows. The strains on the sides of the specimen get "noisy" at the onset of matrix cracking. When the carbon and glass portions are isolated from the side view, this can be done due to the discrete layers, the strains deviate at the point where the side surface strain becomes "noisy". At this instant the carbon layers are becoming fully saturated with cracks and the fiber tow bundles are close to failure.

Earlier, the effective stress-strain response was investigated using the total average strain from the carbon and glass sides. If the strains from each side are broken down and then used to create effective stress-strain responses, we can learn further information about the material. These individual responses can be seen in figure 9. In all the samples the carbon face is always stiffer, showing that through loading further bending is being induced. The compression that was seen in the carbon face on some of the samples can be seen in the stress-strain responses with the fact that there is zero strain or negative strain initially in the response. This causes an overall shift in the response on the carbon side. This is a result of the initially curved specimen being bent and the carbon side experiencing compression upon first loading as the sample straightens out.

Examining the DIC contours in further detail reveals information about the strain localization due to the woven architecture. Figure 10 shows the strain contours occurring on the surface of the specimen. The area of highest strain as seen in the strain contours occurs where the matrix material has begun to crack. The DIC contours are shown on the front of our tension sample, while the back was kept unpainted to watch for any cracks that formed.Comparing the cracks that occur on the back surface to the location of strain concentrations it can be seen that these cracks are causing this strain localization. These cracks occur at the location where the Z-fiber is entering the matrix preform causing an area to be void of fibers and thus causing a matrix pocket, as has been illustrated schematically in figure 10. The location of the Z-fibers causes locally higher level of strains when pulled in tension. This results in small localized areas of strain 


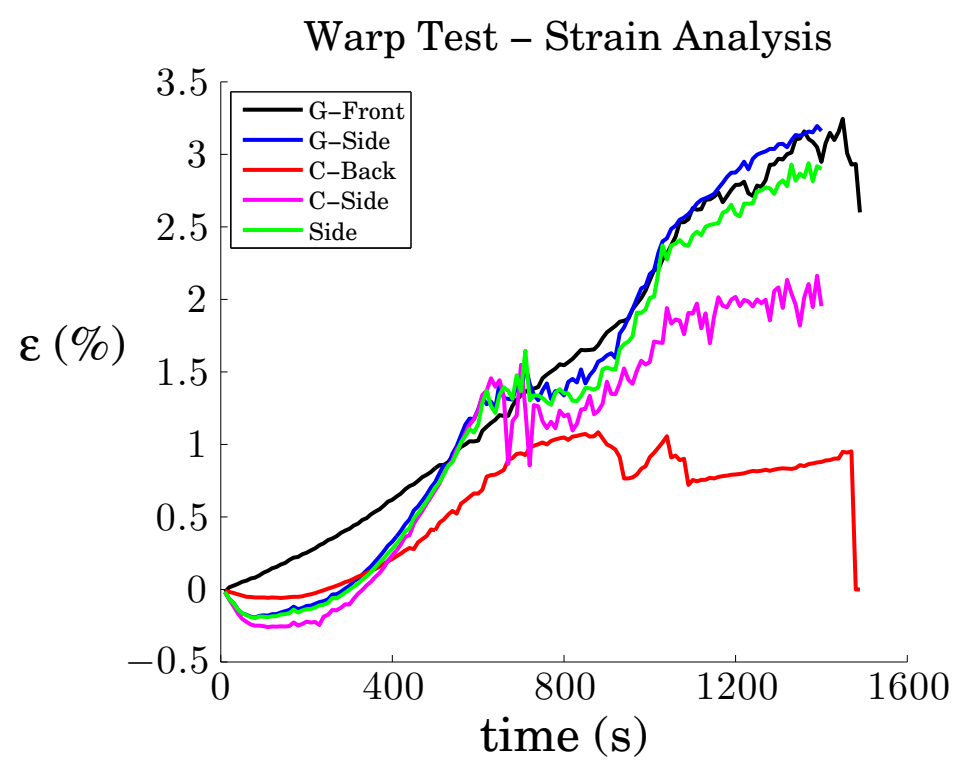

Figure 7. Strain vs. time for composite hybrid samples, comparing the strains that occur on the different surfaces of the material.

that map the surface of the DIC. These will be sites of initial matrix cracking as these tows pull further on the specimens. A similar phenomenon occurs in the weft direction, however, many of the cracks that form on the surface are relatively small and self arrest as they run into a weft tow. They typically occur around the crown of the Z-fiber and form either a single crack in the middle of the crown or two cracks around the outside of the crown.

Moving from the baseline to the unsymmetric hybrid architecture, see figure 11, it is shown that there are very similar strain contour maps on the surface. This image represents the point at which the carbon layers have failed, but the glass layers still carry the load. This is shown in the side view DIC plots which show that the glass still has relatively large strains while the carbon side has relaxed. The warp orientation shows a similar result as compared to the baseline, however, the localization of the strain as the matrix undergoes cracking is much more apparent. Identical behavior is shown in the relaxation in the carbon side of the sample as these fibers have failed first in the material. Thicker unsymmetric material shows similar response as was observed in the thin samples.

The plots corresponding to the symmetric panels, see figure 12, show similar failure characteristics to those observed in both the baseline and the unsymmetric samples. However, in these samples both layers (front and back, like a sandwich panel) of carbon release their strain energy and failure almost simultaneously, forcing the glass layers to carry the remaining load of the material. It is not as easy to see the cracks on the surface of these specimens, however, the DIC strain map is able to show the location of many of the cracks. The weft orientations shows the tows close to the edge are affected by edge effects as can be observed through the vertical cracks that run in these samples. Overall, the response and progressive failure mechanism appear to be similar and thus, will be directly related to material properties of each constituent.

The functionally graded architecture DIC maps are shown in figure 13. The strain contour plots look identical to those seen for the other configurations. Since the material is graded through the thickness (layer to layer discretely), it is difficult to tell how the mixed layers of tows affects the progression of failure. Finite element models, which are under development will shed further light on what is happening on the surface of the material as influenced by the internal functional grading. The surface strains do not reveal any detailed information about the effect of hybridization and its progression of failure. 


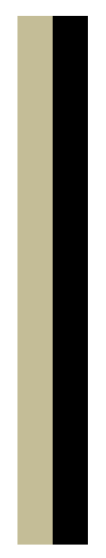

Preform

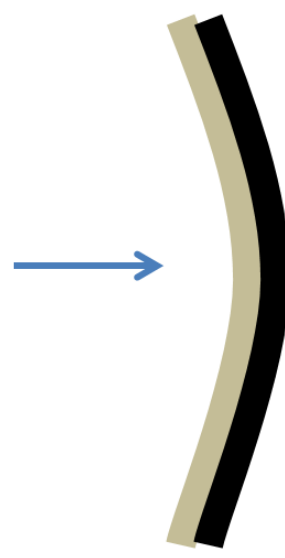

Cured

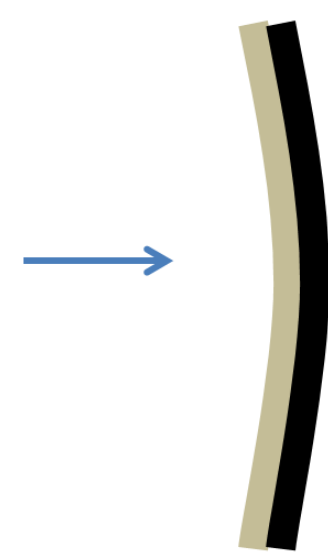

Initial Tension

Figure 8. The process of making the unsymmetrical samples. From these sketches it is seen that the carbon will initially undergo compression as the sample begins to straighten out.

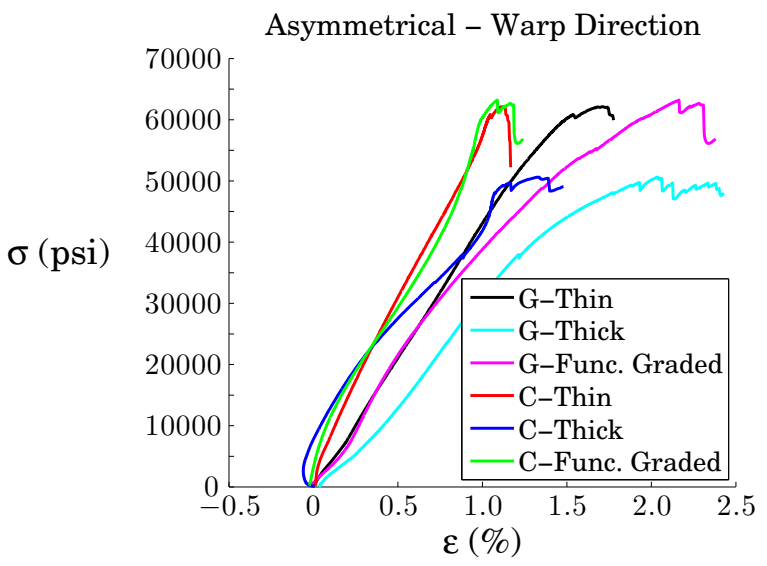

(a) Warp

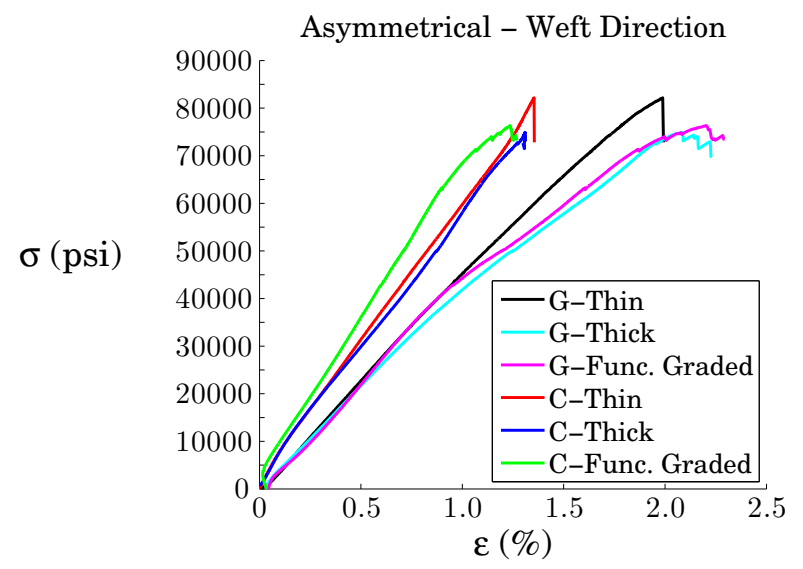

(b) Weft

Figure 9. Comparison of Stress vs. Strain curves for the different unsymmetric panels. G represents the Glass side and $\mathrm{C}$, the Carbon side. 
Nikon Front (Glass)

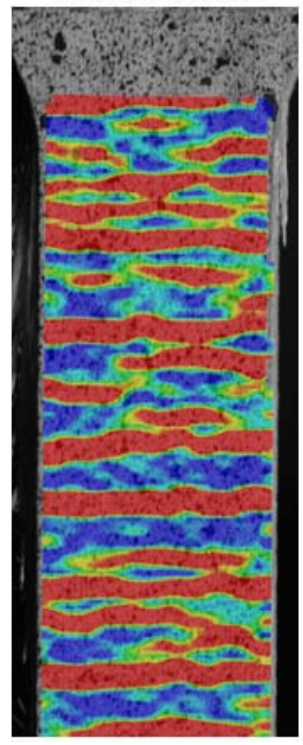

Nikon Front (Glass)
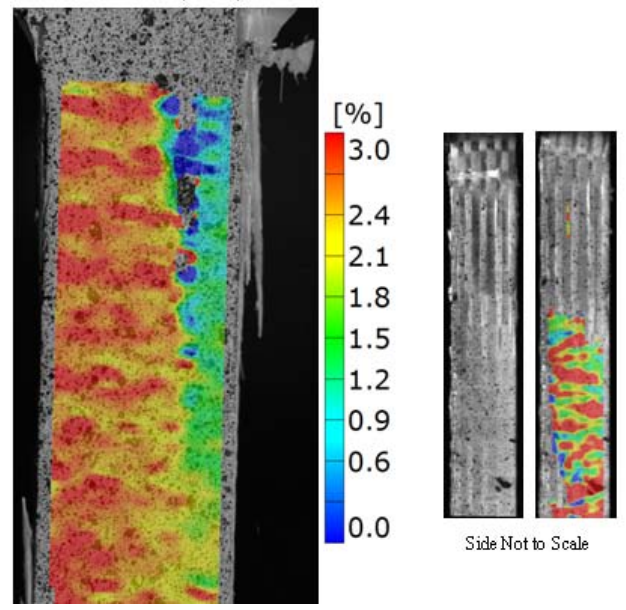

Side Not to Scale

(b) Weft

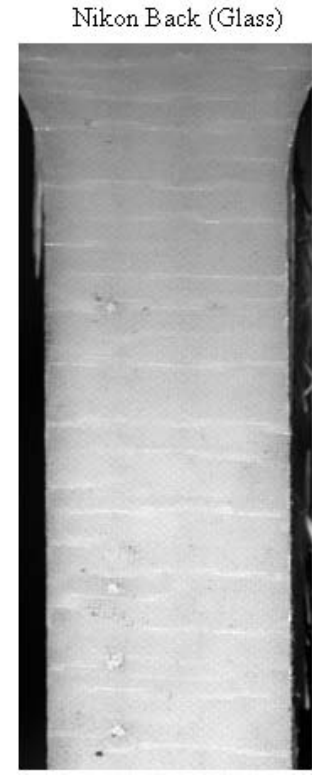

Nikon Back (Glass)
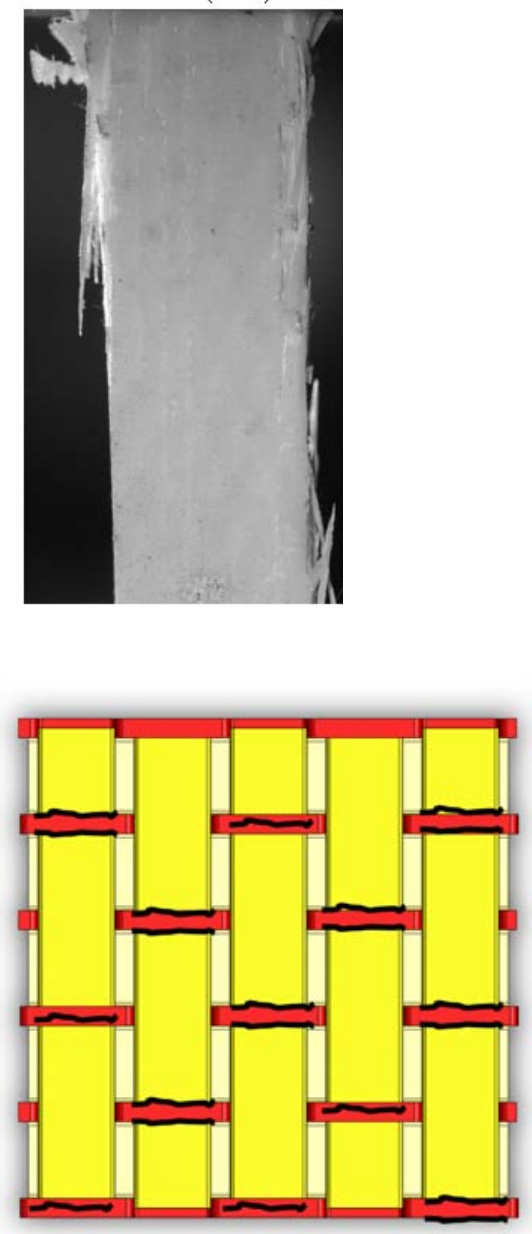

(d) Weft

(c) Warp

Figure 10. Baseline architecture showing strain contour plots overlaid on the samples. The areas of highest strains show the locations of the matrix cracking that is occurring on the surface of the material. Pictorial representations of the crack paths have been presented where the crack paths are represented by the black lines. 

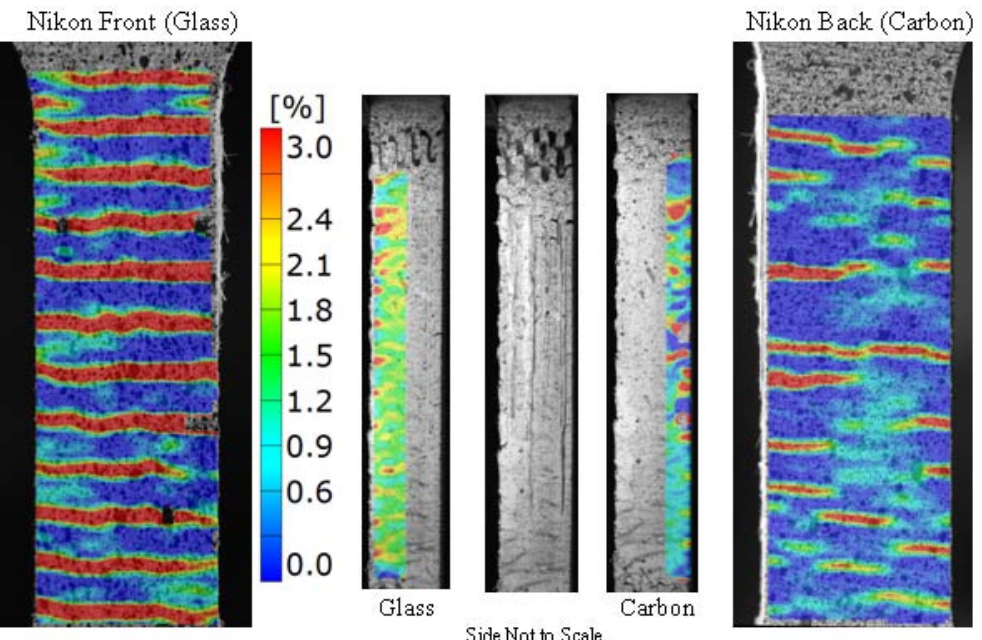

(a) Warp
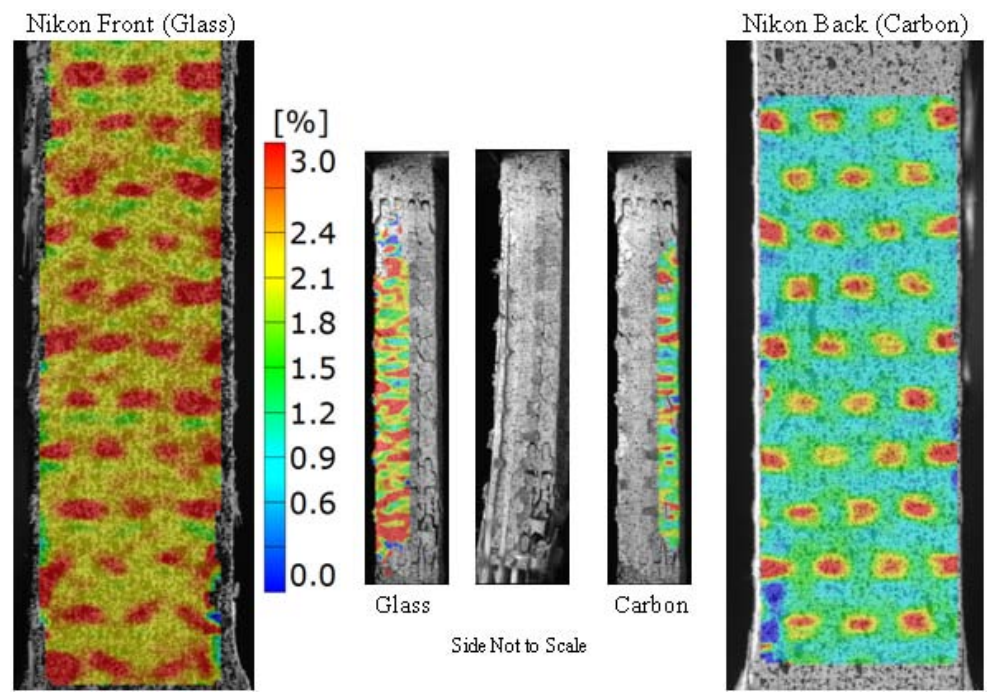

(b) Weft

Figure 11. Thin unsymmetric architecture showing the surface strain contours of the material close to failure of the carbon layers. 

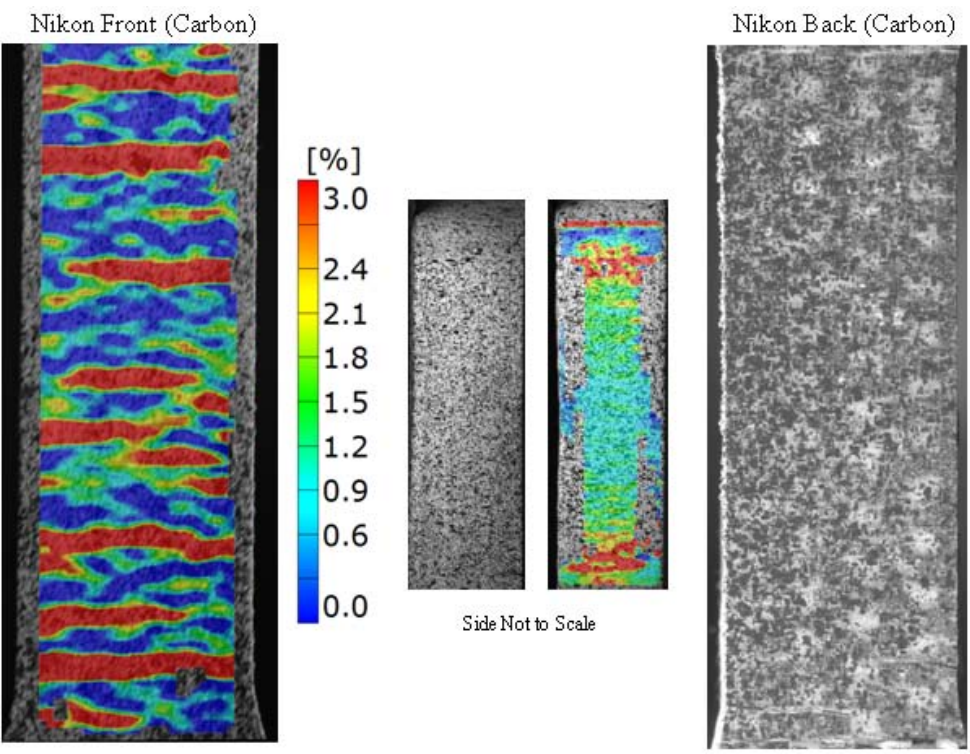

(a) Warp
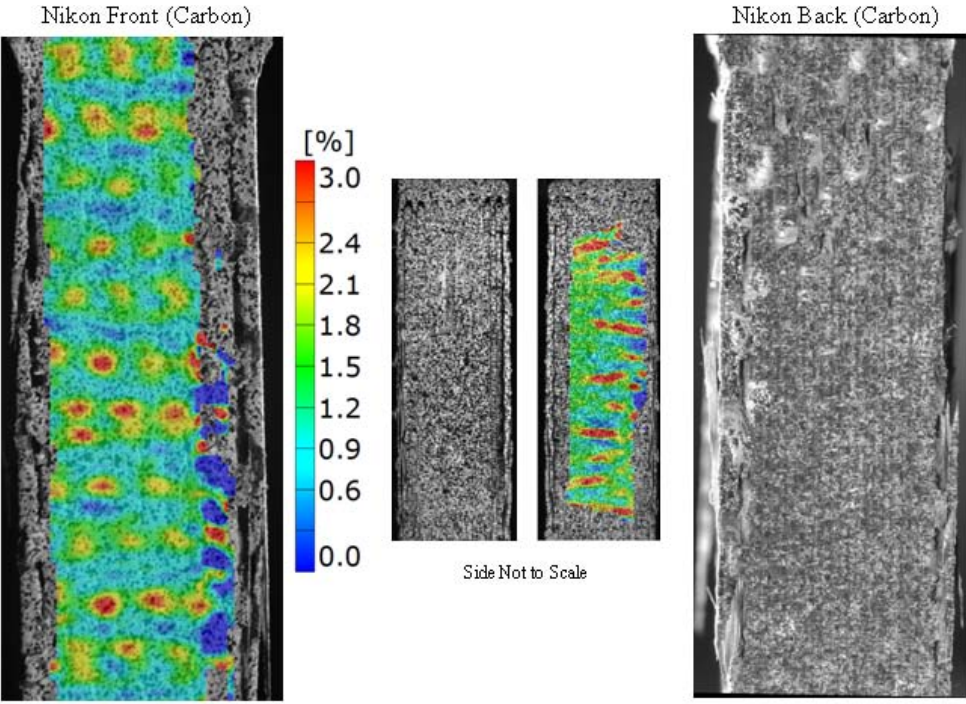

(b) Weft

Figure 12. Symmetric architecture with strain contour plots close to failure of the carbon layers in the material. 

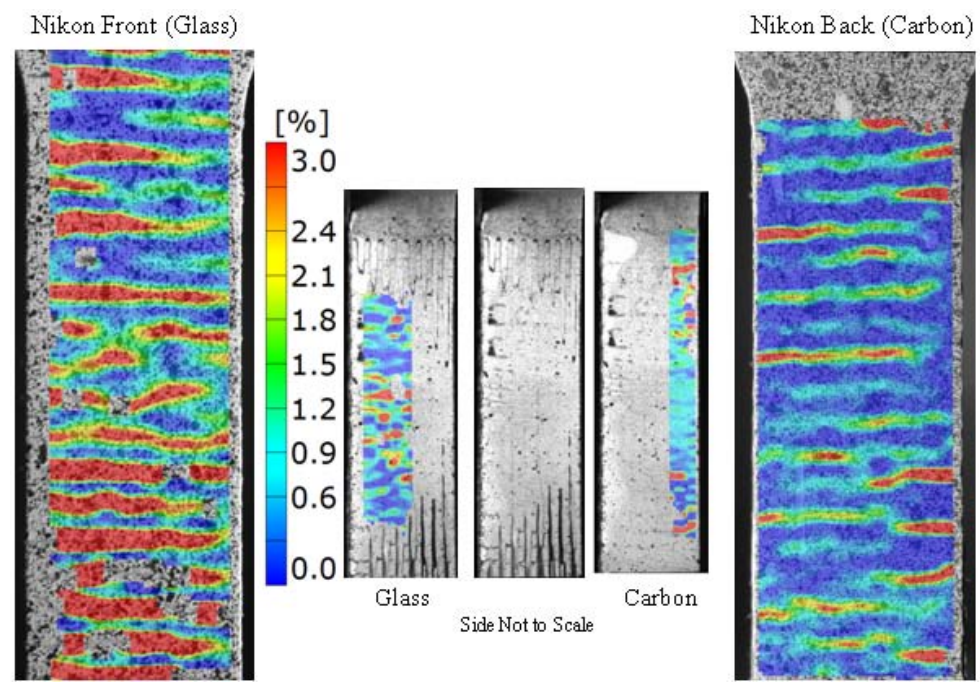

(a) Warp
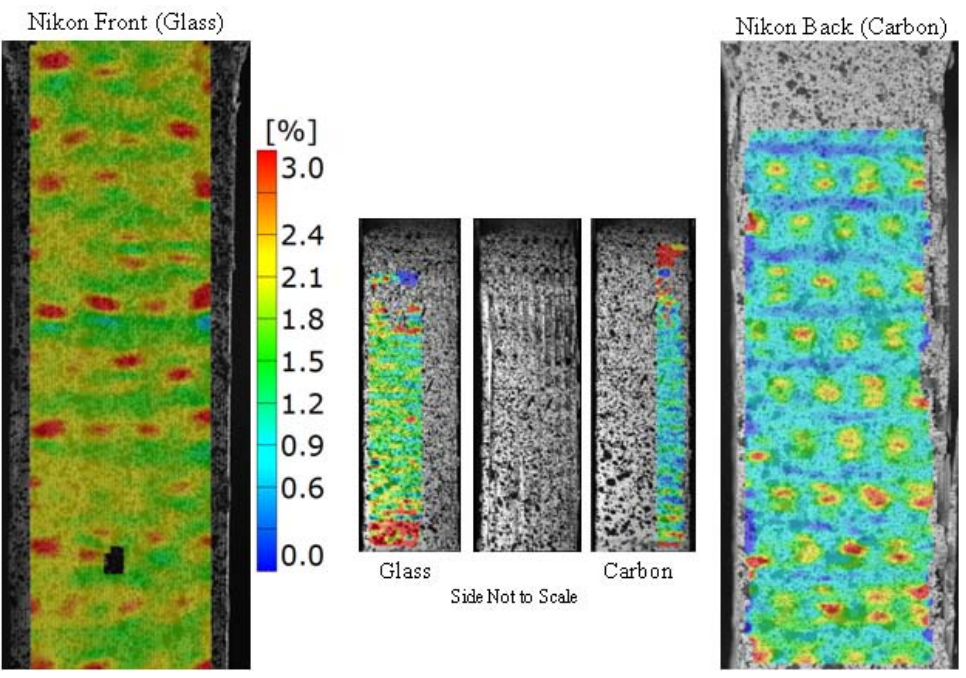

(b) Weft

Figure 13. Strain Contour plots overlaid on the samples to show the locations of the matrix cracking that is occurring on the surface of the material. 


\section{Conclusion}

Hybrid 3D woven textile composites containing different types of fiber tows and different architectures were examined experimentally for their tensile response to determine the effect of hybridization. This effect was investigated to understand the effective stiffness and strength in tension. Results showed that the samples followed a response that was similar to a rule of mixture type prediction for the increase in effective modulus. The strength showed different trends. The pure glass baseline sample exhibited the the highest strength. The additional layers of carbon decreased the overall strength due to the manner by which the load is transferred to the fiber tows. The H3DWC material revealed that there were architecture dependent strains that occurred on the surface of the material. The localization of strains caused matrix micro-cracking between fiber tow bundles which in some orientations will be self arresting. Hybridization, by adding carbon to an existing glass fiber system, appears to provide increases in tensile stiffness but at the expense of a lower ultimate tensile strength.

\section{Acknowledgments}

The authors would like to thank the Army Research Laboratory, Aberdeen Proving Ground, MD, for financial support.

\section{References}

${ }^{1}$ J.P. Quinn, A.T. McIlhagger, and R. McIlhagger. Examination of the failure of 3d woven composites. Composites Part A: Applied Science and Manufacturing, 39(2):273 - 283, 2008.

${ }^{2}$ M. Pankow, A. Salvi, A.M. Waas, C.F. Yen, and S. Ghiorse. Resistance to delamination of 3d woven textile composites evaluated using end notch flexure (enf) tests: Experimental results. Composites Part A: Applied Science and Manufacturing, 42(10):1463 - 1476, 2011.

${ }^{3}$ R. Gerlach, C. R. Siviour, J. Wiegand, and N. Petrinic. In-plane and through-thickness properties, failure modes, damage and delamination in $3 \mathrm{~d}$ woven carbon fibre composites subjected to impact loading. Composites Science and Technology, 72(3):397 - 411, 2012.

${ }^{4}$ S. S. Cheon, T. Seong Lim, and D. G. Lee. Impact energy absorption characteristics of glass fiber hybrid composites. Composite Structures, 46(3):267 - 278, 1999.

${ }^{5}$ G. Marom, E Drukker, A Weinberg, and J. Banbaji. Impact behaviour of carbon/kevlar hybrid composites. Composites, 17(2):150 - 153, 1986.

${ }^{6}$ S. C. Khatri and M. J. Koczak. Thick-section as4-graphite/e-glass/pps hybrid composites: Part i. tensile behavior. Composites Science and Technology, 56(2):181 - 192, 1996.

${ }^{7}$ W. Hufenbach, M. Gude, and C. Ebert. Hybrid 3d-textile reinforced composites with tailored property profiles for crash and impact applications. Composites Science and Technology, 69(9):1422 - 1426, 2009. Special Issue on the 12th European Conference on Composite Materials (ECCM12), organized by the European Society for Composite Materials (ESCM).

${ }^{8}$ C. Heinrich, M. Aldridge, A. S. Wineman, J. Kieffer, A. M. Waas, and K. W. Shahwan. Generation of heat and stress during the cure of polymers used in fiber composites. International Journal of Engineering Science, 53(0):85 - 111, 2012.

${ }^{9}$ B.D. Agarwal, L.J. Broutman, and K. Chandrashekhara. Analysis and performance of Fiber Composites. Wiley, third edition, 2006.

${ }^{10}$ M.C. Saha, S. Nilufar, M. Major, and S. Jeelani. Processing and performance evaluation of hollow microspheres filled epoxy composites. Polymer Composites, 29(3):293-301, 2008.

${ }^{11}$ M.W. Hyer. Stress analysis of fiber reinforced composite materials. In Comprehensive Composite Materials. McGraw Hill, 1997.

\section{Appendix}

The initial curvature of the specimen, due to curing, can be approximately calculated using classical lamination plate theory (CLPT). Thermal strains are produced in the laminates after the composite is cured at an elevated temperature, and its temperature is lowered back to room temperature. These initial strains are due to mismatch in the coefficients of thermal expansion, due to resin shrinkage during curing and because of non-uniform curing (material on the proximities cure first, and additional curing of material leads to solidification of the internally curing material on an already solidified outer layer). Assuming that the entire panel is subjected to a constant temperature change, $\Delta T$,

$$
\{\epsilon\}^{T}=\{\alpha\}_{1} \Delta T
$$

where $\{\alpha\}_{1}$ contains the coefficients of thermal expansion in principal material coordinate. 
For a 2D orthotropic lamina under the condition of plane stress,

$$
\{\alpha\}_{1}=\left\{\begin{array}{c}
\alpha_{L} \\
\alpha_{T} \\
0
\end{array}\right\}
$$

where $\alpha_{L}$ and $\alpha_{T}$ are the coefficients of thermal expansion along longitudinal and transverse directions based on the fiber and matrix directions respectively. It should be noted that the orthotropic material does not exhibit shear strain in the principal material coordinate due to temperature change.

The thermal coefficients for a 2D lamina in the global coordinate system can be obtained by tensor transformation as,

$$
\left\{\begin{array}{c}
\alpha_{x} \\
\alpha_{y} \\
\frac{1}{2} \alpha_{x y}
\end{array}\right\}=\left[\begin{array}{ccc}
\cos ^{2} \theta & \sin ^{2} \theta & -2 \cos \theta \sin \theta \\
\sin ^{2} \theta & \cos ^{2} \theta & 2 \cos \theta \sin \theta \\
\cos \theta \sin \theta & -\cos \theta \sin \theta & \cos ^{2} \theta-\sin ^{2} \theta
\end{array}\right]\left\{\begin{array}{c}
\alpha_{L} \\
\alpha_{T} \\
0
\end{array}\right\}
$$

Therefore the thermal strains can be calculated for each lamina as

$$
\left\{\begin{array}{c}
\epsilon_{x}^{T} \\
\epsilon_{y}^{T} \\
\gamma_{x y}^{T}
\end{array}\right\}=\left\{\begin{array}{c}
\alpha_{x} \Delta T \\
\alpha_{y} \Delta T \\
\alpha_{x y} \Delta T
\end{array}\right\}
$$

The total strains of the material can be written as the sum of the strains due to mechanical loading and thermal strains.

$$
\{\epsilon\}=\{\epsilon\}^{\sigma}+\{\epsilon\}^{T}
$$

From the constitutive relations,

$$
\{\epsilon\}^{\sigma}=[S]\{\sigma\}
$$

where $[S]$ is the compliance matrix. Hence,

$$
\{\epsilon\}=[S]\{\sigma\}+\{\epsilon\}^{T}
$$

Consider the $k^{t h}$ lamina under both mechanical and thermal loads,

$$
\left\{\begin{array}{c}
\epsilon_{x} \\
\epsilon_{y} \\
\gamma_{x y}
\end{array}\right\}=\left[\begin{array}{lll}
S_{11} & S_{12} & S_{16} \\
S_{12} & S_{22} & S_{26} \\
S_{16} & S_{26} & S_{66}
\end{array}\right]_{k}\left\{\begin{array}{c}
\sigma_{x} \\
\sigma_{y} \\
\sigma_{x y}
\end{array}\right\}+\left\{\begin{array}{c}
\epsilon_{x}^{T} \\
\epsilon_{y}^{T} \\
\gamma_{x y}^{T}
\end{array}\right\}_{k}
$$

The stress in the lamina can be obtained by inverting Eq.(8).

$$
\left\{\begin{array}{c}
\sigma_{x} \\
\sigma_{y} \\
\sigma_{x y}
\end{array}\right\}_{k}=\left[\begin{array}{lll}
Q_{11} & Q_{12} & Q_{16} \\
Q_{12} & Q_{22} & Q_{26} \\
Q_{16} & Q_{26} & Q_{66}
\end{array}\right]_{k}\left\{\begin{array}{c}
\epsilon_{x}-\epsilon_{x}^{T} \\
\epsilon_{y}-\epsilon_{y}^{T} \\
\gamma_{x}-\gamma_{x}^{T}
\end{array}\right\}_{k}
$$

Based on the CLPT,

$$
\begin{aligned}
\epsilon_{x} & =\epsilon_{x}^{0}+z k_{x} \\
\epsilon_{y} & =\epsilon_{y}^{0}+z k_{y} \\
\epsilon_{x y} & =\epsilon_{x y}^{0}+z k_{x y}
\end{aligned}
$$


Hence, the laminate stress and moment resultants can be calculated as,

$$
\begin{aligned}
\{N\} & =\sum_{k=1}^{n} \int_{h_{k-1}}^{h_{k}}\{\sigma\}_{k} d z \\
& \sum_{k=1}^{n} \int_{h_{k-1}}^{h_{k}}[Q]_{k}\left[\left\{\epsilon^{0}\right\}+z\{\kappa\}-\{\epsilon\}^{T}\right] d z \\
\{M\} & =\sum_{k=1}^{n} \int_{h_{k-1}}^{h_{k}}\{\sigma\}_{k} z d z \\
& =\sum_{k=1}^{n} \int_{h_{k-1}}^{h_{k}}[Q]_{k}\left[\left\{\epsilon^{0}\right\}+z\{\kappa\}-\{\epsilon\}^{T}\right] z d z
\end{aligned}
$$

The above equations can be written in the matrix form using $A B D$ matrix that is calculated in the CLPT.

$$
\begin{gathered}
\left\{\begin{array}{c}
N_{x} \\
N_{y} \\
N_{x y}
\end{array}\right\}=\left[\begin{array}{lll}
A_{11} & A_{12} & A_{16} \\
A_{12} & A_{22} & A_{26} \\
A_{16} & A_{26} & A_{66}
\end{array}\right]\left\{\begin{array}{c}
\epsilon_{x}^{0} \\
\epsilon_{y}^{0} \\
\gamma_{x y}^{0}
\end{array}\right\}+\left[\begin{array}{lll}
B_{11} & B_{12} & B_{16} \\
B_{12} & B_{22} & B_{26} \\
B_{16} & B_{26} & B_{66}
\end{array}\right]\left\{\begin{array}{c}
\kappa_{x} \\
\kappa_{y} \\
\kappa_{x y}
\end{array}\right\}-\left\{\begin{array}{c}
N_{x}^{T} \\
N_{y}^{T} \\
N_{x y}^{T}
\end{array}\right\} \\
\left\{\begin{array}{c}
M_{x} \\
M_{y} \\
M_{x y}
\end{array}\right\}=\left[\begin{array}{lll}
B_{11} & B_{12} & B_{16} \\
B_{12} & B_{22} & B_{26} \\
B_{16} & B_{26} & B_{66}
\end{array}\right]\left\{\begin{array}{c}
\epsilon_{x}^{0} \\
\epsilon_{y}^{0} \\
\gamma_{x y}^{0}
\end{array}\right\}+\left[\begin{array}{lll}
D_{11} & D_{12} & D_{16} \\
D_{12} & D_{22} & D_{26} \\
D_{16} & D_{26} & D_{66}
\end{array}\right]\left\{\begin{array}{c}
\kappa_{x} \\
\kappa_{y} \\
\kappa_{x y}
\end{array}\right\}-\left\{\begin{array}{c}
M_{x}^{T} \\
M_{y}^{T} \\
M_{x y}^{T}
\end{array}\right\}
\end{gathered}
$$

where

$$
\begin{gathered}
\left\{\begin{array}{c}
N_{x}^{T} \\
N_{y}^{T} \\
N_{x y}^{T}
\end{array}\right\}=\sum_{k=1}^{n}\left[\begin{array}{lll}
Q_{11} & Q_{12} & Q_{16} \\
Q_{12} & Q_{22} & Q_{26} \\
Q_{16} & Q_{26} & Q_{66}
\end{array}\right]_{k}\left\{\begin{array}{c}
\alpha_{x} \\
\alpha_{y} \\
\alpha_{x y}
\end{array}\right\} \Delta T\left(h_{k}-h_{k-1}\right) \\
\left\{\begin{array}{l}
M_{x}^{T} \\
M_{y}^{T} \\
M_{x y}^{T}
\end{array}\right\}=\frac{1}{2} \sum_{k=1}^{n}\left[\begin{array}{lll}
Q_{11} & Q_{12} & Q_{16} \\
Q_{12} & Q_{22} & Q_{26} \\
Q_{16} & Q_{26} & Q_{66}
\end{array}\right]_{k}\left\{\begin{array}{c}
\alpha_{x} \\
\alpha_{y} \\
\alpha_{x y}
\end{array}\right\} \Delta T\left(h_{k}^{2}-h_{k-1}^{2}\right)
\end{gathered}
$$

Supposing that

$$
\begin{aligned}
& \left\{\begin{array}{c}
\bar{N}_{x} \\
\bar{N}_{y} \\
\bar{N}_{x y}
\end{array}\right\}=\left\{\begin{array}{c}
N_{x} \\
N_{y} \\
N_{x y}
\end{array}\right\}+\left\{\begin{array}{c}
N_{x}^{T} \\
N_{y}^{T} \\
N_{x y}^{T}
\end{array}\right\} \\
& \left\{\begin{array}{c}
\bar{M}_{x} \\
\bar{M}_{y} \\
\bar{M}_{x y}
\end{array}\right\}=\left\{\begin{array}{c}
M_{x} \\
M_{y} \\
M_{x y}
\end{array}\right\}+\left\{\begin{array}{c}
M_{x}^{T} \\
M_{y}^{T} \\
M_{x y}^{T}
\end{array}\right\}
\end{aligned}
$$

Then

$$
\left\{\begin{array}{l}
\bar{N} \\
\bar{M}
\end{array}\right\}=\left[\begin{array}{ll}
A & B \\
B & D
\end{array}\right]\left\{\begin{array}{l}
\epsilon^{0} \\
\kappa
\end{array}\right\}
$$

Assuming that there is no mechanical load applied on the material during the curing process, then

$$
N_{x}=N_{y}=N_{x y}=M_{x}=M_{y}=M_{x y}=0
$$

and

$$
\left\{\begin{array}{l}
\bar{N} \\
\bar{M}
\end{array}\right\}=\left\{\begin{array}{l}
N^{T} \\
M^{T}
\end{array}\right\}=\left[\begin{array}{ll}
A & B \\
B & D
\end{array}\right]\left\{\begin{array}{c}
\epsilon^{0} \\
\kappa
\end{array}\right\}
$$


Hence the in-plane strains and curvatures can be calculated by inverting Eq.(19)

$$
\left\{\begin{array}{l}
\epsilon^{0} \\
\kappa
\end{array}\right\}=\left[\begin{array}{ll}
A & B \\
B & D
\end{array}\right]^{-1}\left\{\begin{array}{l}
N^{T} \\
M^{T}
\end{array}\right\}
$$

where $\left[N^{T}\right]$ and $\left[M^{T}\right]$ can be calculated using Eq.(15) and (16).

The overall thickness of the unsymmetric laminate was estimated at $9 \mathrm{~mm}$ based on experimental measurements, discussed earlier. The temperature is reduced from oven to room temperature, which is equivalent to $-190^{\circ} \mathrm{F}$ change in temperature. Using lamina properties given in table 5 , as an initial estimate, the curvatures of the panel have been calculated. The calculations estimate a curvature of 292 in. for the weft direction and $218 \mathrm{in}$. for the warp direction. These estimates do not account for chemical shrinkage and cure kinetics,$-^{8}$ and also assumes a constant, $\Delta T$.

Using CLPT calculations one can also find the effective modulus of the material. This is done after expanding the terms and setting all in plane forces equal to zero except in the direction of loading.

$$
\left\{\begin{array}{c}
\bar{N}_{x} \\
\bar{N}_{y} \\
\bar{N}_{x y} \\
\bar{M}_{x} \\
\bar{M}_{y} \\
\bar{M}_{x y}
\end{array}\right\} \rightarrow\left\{\begin{array}{c}
N_{x}+N_{x}^{T} \\
0+N_{y}^{T} \\
0+N_{x y}^{T} \\
0+M_{x}^{T} \\
0+M_{y}^{T} \\
0+M_{x y}^{T}
\end{array}\right\}=\left[\begin{array}{ll}
A & B \\
B & D
\end{array}\right]\left\{\begin{array}{c}
\epsilon^{0} \\
k
\end{array}\right\}
$$

Then by solving this system of equations for $N_{x}$ in terms of $\epsilon_{x}$ allows us to find the effective modulus of the material. Based on these calculations the effective modulus of the material is 4.52 msi for the warp and $5.46 \mathrm{msi}$ for the weft. These compare very well to those measured experimentally which were $4.61 \mathrm{msi}$ in the warp and $5.00 \mathrm{msi}$ in the weft, lending confidence to the estimated CLPT, ABBD matrices.

Table 5. Material Properties for typical unidirectional laminates ${ }^{9}$

\begin{tabular}{ccccccccc} 
& $\begin{array}{c}E_{1} \\
\mathrm{msi}\end{array}$ & $\begin{array}{c}E_{2} \\
\mathrm{msi}\end{array}$ & $\begin{array}{c}\nu_{12} \\
-\end{array}$ & $\begin{array}{c}\nu_{23} \\
-\end{array}$ & $\begin{array}{c}G_{12} \\
\mathrm{msi}\end{array}$ & $\begin{array}{c}G_{23} \\
\mathrm{msi}\end{array}$ & $\frac{\mu}{{ }^{\circ} \mathrm{F}}$ & $\frac{\mu}{{ }^{\circ} \mathrm{F}}$ \\
\hline Carbon & 21.5 & 1.46 & 0.3 & 0.59 & 0.81 & 0.46 & -0.44 & 16 \\
\hline Glass & 6.31 & 1.67 & 0.27 & 0.40 & 0.50 & 0.60 & 3.8 & 16 \\
\hline
\end{tabular}

Models made using the finite element software package ABAQUS were also used evaluate to determine the effective curvature. Two levels of modeling were evaluated. The first is based on the built in composite laminate model. The laminate sequence was based on the nine total layers of warp and weft fibers, five weft fiber layers and four warp fiber layers. Each layer is modeled with its own individual orientation. The computational model predicts a curvature of $236 \mathrm{in}$. in the weft direction and 205 in. in the warp direction, which is similar to that calculated by CLPT.

A refined model was then investigated, which broke down each individual layer into its constituents. Each effective layer is modeled as a shell which is broken down discretely to have fiber tow bundles and matrix tow bundles, see figure 14. Each of the layers was then tied together to prevent sliding between the layers. The coefficient of thermal expansion for SC-15 matrix material is $47.6 \frac{\mu m}{m^{\circ} \mathrm{F}} \cdot{ }^{10}$ A thermal load was then applied and the resulting curvature was found in the warp direction. This showed a radius of curvature of $184 \mathrm{in.}$ in the weft direction and $160 \mathrm{in}$. in the warp direction. This more refined model shows the pockets of matrix have some effect on the curvature, however, the estimates over-predict the amount of initial bending, compared to CLPT and measurements.

The coefficients of thermal expansion can be found for an individual fiber tow bundle. The details are shown in ${ }^{11}$.

$$
\alpha_{1}=\frac{\left(\alpha_{1}^{f} E_{1}^{f}-\alpha^{m} E^{m}\right) V^{f}+\alpha^{m} E^{m}}{\left(E_{1}^{f}-E^{m}\right) V^{f}+E^{m}}
$$




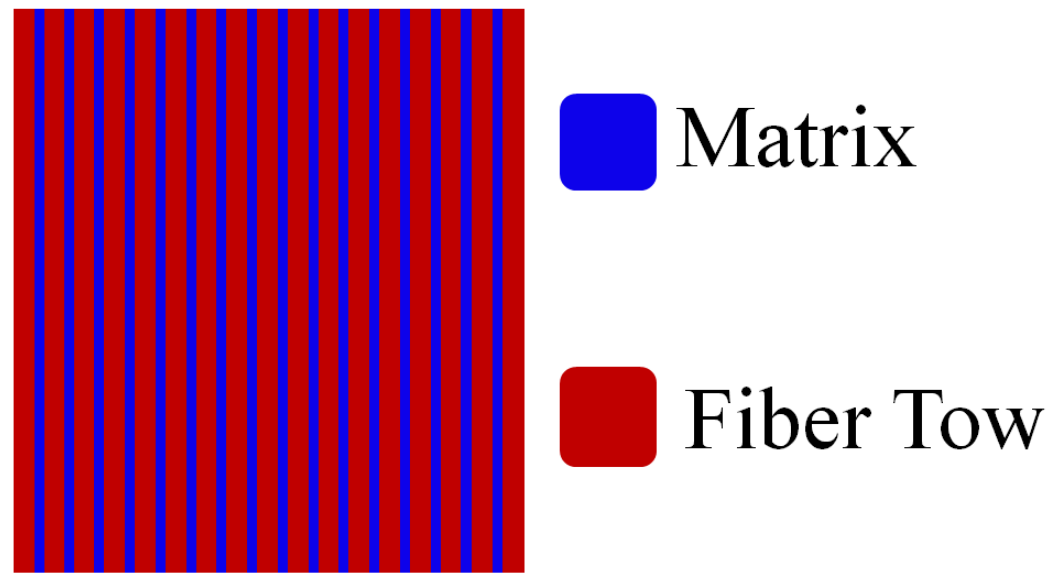

Figure 14. Discrete Fiber tow bundle shell model

$$
\alpha_{2}=\alpha_{2}^{f} V^{f}+\alpha^{m}\left(1-V^{f}\right)+\frac{E_{1}^{f} \nu^{m}-E^{m} \nu_{12}^{f}}{E_{1}}\left(\alpha^{m}-\alpha_{1}^{f}\right)\left(1-V^{f}\right) V^{f}
$$

Where $E_{1}$ is the fiber tow bundle modulus, which can be approximated by a rule of mixture calculation or the Concentric cylinder model calculations. The properties for the individual constituents are given in table 6 .

Table 6. Material Characteristics for fibers and matrix used

\begin{tabular}{ccccc} 
& $\begin{array}{c}E_{1} \\
\mathrm{msi}\end{array}$ & $\begin{array}{c}\nu_{12} \\
-\end{array}$ & $\begin{array}{c}\alpha_{1} \\
\frac{\mu m}{m^{\circ} \mathrm{F}}\end{array}$ & $\begin{array}{c}\alpha_{2} \\
\frac{\mu m}{m^{\circ} \mathrm{F}}\end{array}$ \\
\hline Carbon & 40.03 & 0.2 & -0.2 & 2.83 \\
\hline Glass & 16.56 & 0.22 & 0.89 & \\
\hline SC-15 & 0.360 & 0.35 & 47.6 & \\
\hline
\end{tabular}

From these, the effective coefficients of thermal expansion can be calculated to determine the resulting expansion coefficients for fiber tow bundles. The carbon tows have a fiber volume fraction of $60 \%$ and the glass tows have $55 \%$. The coefficients of thermal expansion were found and reported in table 7 , these values have similar trends to those used from pre-preg material (see table 5).

Table 7. Coefficients of thermal expansion

\begin{tabular}{ccc} 
& $\begin{array}{c}\alpha_{1} \\
\frac{\mu m}{m^{\circ} \mathrm{F}}\end{array}$ & $\begin{array}{c}\alpha_{2} \\
\frac{\mu m}{m^{\circ} \mathrm{F}}\end{array}$ \\
\hline Carbon & 0.074 & 26.78 \\
\hline Glass & 1.69 & 28.76 \\
\hline
\end{tabular}

